\title{
LA FARINE LACTÉE
}

\author{
par \\ M. A. CUÉNOT \\ Ingénieur Agronome
}

Diplômé de la Section d'Etudes supérieures des Industriess du lait

\section{A. Origine}

La fabrication de la farine lactée est assurée, dans la grande majorité des cas, par des fabricants de lait concentré.

Cette observation est évidemment explicable par des raisons techniques. En effet, les condenseurs, faisant également du lait en poudre, ont à leur disposition, d'une part le lait, d'autre part une grande partie des appareils qui leur sont nécessaires à la fabrication industrielle de la farine lactée.

Mais l'industrialisation de la farine lactée paraît avoir son origine dans la satisfaction de buts essentiellement commerciaux.

En effet, ce produit est un des principaux aliments des enfants du deuxième âge, e'est-à-dire âgés d'au moins six mois, alors que le lait constitue la seule et unique nourriture des enfants du premier âge (de 0 à 6 mois). Aussi les fabricants de lait condensé, dont la clientèle est avant tout cómposée des mères de famille, ont-ils eu l'idée d'offrir à leurs clientes, les bouillies dont les enfants font, à partir du sixième mois, une si grande consommation.

Il s'agissait, pour les fabricants de lait condensé, de mettre à la disposition des mères de famille, un produit offrant à la fois toutes les garanties hygiéniques désirables et dont la présentation et l'utilisation rappellent celles du lait condensé, en permettant notamment de se servir des biberons. De plus, l'aliment du deuxième âge doit être d'un usage aussi commode que possible, simple et facile à préparer : par adjonction d'eau chaude ou froide, et ébullition.

Commercialement parlant, les produits infantiles obligent les jeunes mères de famille à rester fidèle à la marque de lait condensé qu'elles ont choisie. Cette fidélité, encouragée par une publicité judicieuse mettant en valeur les excellents résultats des produits lactés utilisés, est ainsi prolongée suffisamment longtemps pour inciter les mamans à utiliser, à la naissance d'un nouveau bébé, toujours la même marque, sans la moindre solution de continuité.

C'est done surtout le renforcement de leur position commerciale qui a incité les condenseurs à proposer au public, en plus du lait condensé ordinaire, des farines lactées, puis des farines composées, lactées ou non, permettant de "suivre " l'enfant jusqu'à l'âge où normalement une nouvelle naissance au foyer assure la 
continuité de consommation que les industriels ont toujours souhaité,

Enfin, la farine lactée, aliment riche, nourrissant et facilement digestible, intéresse non seulement les bébés, mais encore les adolescents, les vieillards, les malades. Et cette clientèle, si elle n'est pas la principale, représente cependant un débouché intéressant.

La meilleure façon d'atteindre ces consommateurs, commed'ailleurs les mères de famille, est le canal médical, et par voie de conséquence, les pharmaciens. Aussi, est-ce directement par le corps médical, beaucoup plus que par la grande publicité, que les condenseurs prospectent, avec les meilleurs résultats, leur clientèle.

\section{B. Composition}

La concurrence a provoqué l'apparition de nombreuses variétés de farines infantiles dont la formule diffère avec les fabricants. Celle-ci est d'ailleurs indiquée sur les boîtes, plus ou moins sommairement, mais toujours très exactement.

Nous n'allons pas nous attacher à un classement de tous ces produits, seules les farines lactées nous retiendront.

Que s'agit-il d'offrir au juste au public ? L'enfant du deuxième âge, de six mois environ, est assez grand pour assimiler une nourriture un peu moins facile à digérer que le lait et le sucre. L'élément type, le moins cher, est celui du pain : l'amidon. Il a une grande valeur énergétique, est facile à trouver et est tout de même moins onéreux. Par conséquent, au lait et au sucre, il sera ajouté de la farine.

Afin d'équilibrer la ration, des matières minérales, calciques, si indispensables à la formation des os, complèteront le mélange. Un parfum (vanille, malt, cacao) rendra finalement la farine lactée agréable à ingérer, tout en augmentant éventuellement sa digestibilité.

Cette concession au goût du consommateur lui permet ainsi de choisir le produit qu'il préfère.

En résumé, les farines lactées seront composées principalement de lait, de sucre et de farine auxquels sont ajoutés, à titre accessoire, un parfum et des phosphates de chaux.

La composition la plus simple et la plus courante, en négligeant les éléments accessoires, sera : $50 \%$ farine, $25 \%$ lait, $25 \%$ sucre.

Cette proportion sera celle également de l'extrait sec, car la farine lactée est une poudre pratiquement sèche. Ceci représente le mélange suivant, l'extrait sec du lait étant 125 grammes par litre : 


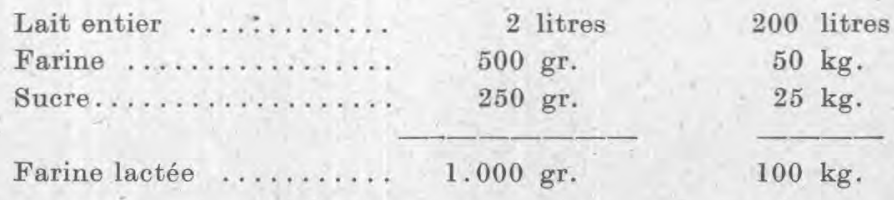

A la différence du lait concentré, la composition du produit fini n'aura pas besoin d'être rigoureusement constante, ear la farine lactée est un aliment destiné à des organismes déjà tolérants à de légères différences de composition. Autrement dit, le mélange au départ, qui servira à faire le produit fabriqué, n'aura pas besoin d'être ajusté suivant la composition de chaque livraison de farine ou suivant la saison pour le lait. Par conséquent, la formule 1/2, $1 / 4,1 / 4$, sera valable toute l'année.

\section{Fabrication}

Ceci posé, voyons la fabrication industrielle de la farine lactée. Le principe consiste à préparer une bouillie de la composition prévue, de la sécher et de la mettre en boîte.

Pratiquement, la fabrication est plus complexe, car le séchage d'un liquide sucré se fait mal, ce qui oblige à ajouter le sucre à la fin de la préparation. De plus, il est bon que la farine, pour être plus assimilable et contenir moins d'eau (conservation) soit rôtie. Avant la mise en boîte, le produit doit donc être refroidi.

Les opérations sont done les suivantes :

\section{Pétrissage.}

Un mélange de la totalité du lait, de la totalité de la farine nécessaire (dont une petite partie peut être maltée) et d'une faible partie du sucre est fait dans un pétrin. Le mélange est bien brassé et chauffé jusqu'à homogénéisation complète.

\section{Séchage.}

La "soupe " ainsi obtenue est pompée et stockée dans un bac situé au-dessus d'un séchoir à cylindres Hatmacker. Elle redescend sur le séchoir qui réduit son humidité de $5 \%$ au maximum. Le film ainsi obtenu est recueilli dans de grandes caisses, à la sortie du séchoir.

\section{Rôtissage.}

Le film est mélangé au reste du sucre, homogénéisé et rôti au four continu, dans des grands plateaux.

La fariue sort du four grillée et les plateaux sont mis à refroidir dans une pièce aérée, sur des claies.

\section{Remplissage.}

Enfin, la farine grillée et refroidie est passée au broyeur. La 
poudre fine est dirigée par une chaîne à godets aux remplisseuses où elle est mise en boîte. Les boîtes sont ensuite étiquetées et mises en caisses.

La capacité des machines employées dépend des usines et des possibilités de production. Mais toute la marche de la fabrication, notamment les mélanges, est suivie au moyen des quantités de farines lactées à obtenir finalement.

Voici, à titre indicatif, une fabrication telle qu'elle a été suivie dans une usine.

Dans la journée, il a été procédé à quatre pétrissées qui ont donné des soupes qui se sont mélangées dans un bac en charge sur le Hatmacker. Douze séchages ont été faits et le film obtenu, auquel il a été ajouté le complément du sucre, a fait l'objet de douze rôtissages qui, après broyage et empaquetage, ont permis d'obtenir la farine lactée.

Les pétrissages sont les suivants :

JOURNÉE DU $\ldots$ (UNITÉ : KG.)

\begin{tabular}{|c|c|c|c|c|c|c|c|}
\hline No de pétrissée & $\begin{array}{c}\text { Heure } \\
\text { de début }\end{array}$ & $\begin{array}{l}\text { Farine } \\
\text { maltée }\end{array}$ & $\begin{array}{c}\text { Farine } \\
\text { blan- } \\
\text { che }\end{array}$ & Suere & $\begin{array}{l}\text { Lait } \\
\text { frais }\end{array}$ & $\begin{array}{l}\text { Lait en } \\
\text { poudre } \\
\text { écrémé }\end{array}$ & Crème \\
\hline$\ldots \ldots \ldots$ & $5 \mathrm{~h} .30$ & 80 & 267 & 42 & 700 & 45 & 44,4 \\
\hline $2 \ldots \ldots \ldots \ldots$ & 10 h. 50 & 80 & 267 & 42 & 700 & 45 & 44,4 \\
\hline $3 \ldots \ldots \ldots \ldots$ & $16 \mathrm{~h} .50$ & 80 & 267 & 42 & 700 & 45 & 44,4 \\
\hline $4 \ldots \ldots \ldots \ldots$ & 23 h. 30 & 80 & 267 & 42 & 700 & 45 & 44,4 \\
\hline Total.. & & 13 & & 168 & 2.800 & 180 & 177,6 \\
\hline
\end{tabular}

Les douze séchages, un toute les deux heures environ, ont été les suivants (voir tableau ci-après).

Les rôtissages se sont échelonnés de 13 heures le jour au lendemain à 11 heures.

\section{Technique}

Le contrôle technique de la fabrication est fondé uniquement sur le respect de la formule: $1 / 4,1 / 4,1 / 2$.

\section{Proportion de la farine.}

Un pétrin dont la capacité est de 1.700 litres peut produire 600 kilogrammes de farine lactée par pétrissée.

Cette quantité est purement expérimentale et peut être modifiée, mais 600 kilogrammes est un lchiffre rond, commode et utilisé pour le travail courant. 
JOURNEE DU .... ET NUIT SUIVANTE

\begin{tabular}{|c|c|c|c|c|}
\hline No du séchage & Heure & $\begin{array}{l}\text { Film } \\
\text { obtenu }\end{array}$ & $\begin{array}{l}\text { Sucre } \\
\text { ajouté }\end{array}$ & Observations \\
\hline $1 \ldots \ldots$ & 8 h. 30 & 170 & 36 & \\
\hline $2 \ldots \ldots \ldots$ & 10 h. 20 & 170 & 36 & \\
\hline $3 \ldots \ldots \ldots$ & 12 heures & 170 & 36 & \\
\hline $4 \ldots \ldots \ldots$ & $13 \mathrm{~h} .45$ & 170 & 36 & \\
\hline $5 \ldots \ldots \ldots$ & 15 h. 30 & 170 & 36 & \\
\hline $6 \ldots \ldots$ & 17 h. 25 & 170 & 36 & \\
\hline $7 \ldots \ldots$ & 19 h. 10 & $\cdot 170$ & 36 & \\
\hline $8 \ldots \ldots$, & 20 h. 50 & 170 & 36 & \\
\hline $9 \ldots \ldots \ldots$ & 22 h. 40 & 170 & 36 & \\
\hline $10 \ldots \ldots$ & 24 h. 20 & 170 & 36 & \\
\hline $11 \ldots \ldots$ & 2 heures & 170 & 36 & \\
\hline $12 \ldots \ldots \ldots$ & 4 heures & 157 & 33 & reste du bac \\
\hline 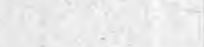 & & 2.027 & 419 & \\
\hline
\end{tabular}

600 kilogrammes de farine lactée représentent 300 kilogrammes d'extrait sec de farine. La farine dosant environ 15 à $16 \%$ d'humidité, il faut en mettre, en tenant compte de $3 \%$ de perte :

$$
\frac{300 \times 100}{87}=347 \mathrm{~kg} \text {. dans le pétrin, }
$$

soit 80 kilogrammes de farine maltée (proportion modifiable à volonté) et 267 kilogrammes de farine blanche.

\section{Proportion du sucre.}

Dans 600 kilogrammes de farine lactée, il doit y avoir :

$$
\frac{600}{4}=150 \mathrm{~kg} \text {. de sucre. }
$$

Pour que le séchage au Hatmacker se fasse bien, la proportion de 42 kilogrammes de sucre par pétrissée est la meilleure. Il reste done 108 kilogrammes de sucre à ajouter, par pétrissée, ensuite.

Or, par séchage, il a été obtenu 170 kilogrammes de film à $5 \%$ d'humidité.

Comme il faut 12 séchages pour 4 pétrissages, un séchage correspond à :

$$
\frac{600 \times 4}{12}=\frac{600}{3}=200 \mathrm{~kg} \text {. de farine lactée. }
$$

Il faudra donc compléter les 170 kilogrammes de film obtenu à chaque séchage par : 


$$
\frac{108}{3}=36 \mathrm{~kg} \text {, de sucre. }
$$

Cela fera 206 kilogrammes de farine lactée qui, à $3 \%$ d'humidité, correspondent à un extrait sec de 200 kilogrammes.

\section{Proportion du lait.}

Si les proportions de farine et de sucre à mettre en œuvre sont invariables et peuvent être fixées au personnel une fois pour toutes, le lait nécessaire à la fabrication est préparé chaque jour et envoyé à l'avance dans un bac muni d'un agitateur, en charge sur le pétrin.

a) But à atteindre.

Nous avons vu que le lait entrait dans la proportion de $25 \%$ dans la composition de la farine lactée, et que 200 litres ou 200 kilogrammès de lait étaient nécessaires pour fabriquer 100 kilogrammes de farine lactée.

Ces 200 kilogrammes de lait apporteront 25 kilogrammes d'extrait sec composé lui-méme de 7 kilogrammes de matière grasse et de 18 kilogrammes d'extrait sec non gras correspondant à 3 gr. 5 de matière grasse (M.G.) et 90 grammes d'extrait see non gras (E.S.N.G.) par kilogramme (ou litre) de lait.

Par conséquent, pour une pétrissée de 600 kilogrammes de farine lactée, le lait à ajouter à la farine et au sucre devra apporter :

réparti en :

$$
600 \times 25 \%=150 \mathrm{~kg} \text {. d'extrait see, }
$$

$$
600 \times 7 \%=42 \mathrm{~kg} \text {. de matière grasse, }
$$

$$
600 \times 18 \%=\frac{108 \mathrm{~kg} \text {. d'extrait sec non gras. }}{150 \mathrm{~kg} . \text { de lait sec. }}
$$

b) Eléments dont dispose le laboratoire.

L'usine de lait condensé qui entreprend la fabrication de farine lactée dispose de lait sous quantités de formes :

Lait de ramassage, provenant de la collecte.

Lait standard, pris à la condenserie.

Lait écrémé et crème, provenant de la!̣ beurrerie et de la standardisation.

Lait concentré sucré ou non, provenant de boîtes couleuses ou de fabrication anormale.

Lait en poudre entier ou écrémé : lait stocké sous cette forme ou provenant d'apports extérieurs.

La préparation de la farine lactée avec ses pétrissage, séchage et rôtissage est la fabrication qui absorbe le plus commodément tous ces excédents de lait que l'on peut trouver dans une condenserie.

Aussi, il est essentiel que le labora toire de l'usine prépare jour- 
nellement le lait destiné à la fabrication de la farine lactée, suivant les éléments dont il dispose, éléments qui varient tous les jours en quantités et en qualité.

Des tables peuvent être établies à l'avance. Peut-être les calculs doivent être confinmés par la pratique (pertes en cours de fabrication) mais néanmoins, il est possible de déterminer les quantités de lait à mettre en œuvre. C'est ce que nous allons faire dans l'exemple choisi.

Nous allons poser à l'avance les compositions des éléments dont nous disposons.

Matière premièré (poids dans un kilogramme) :

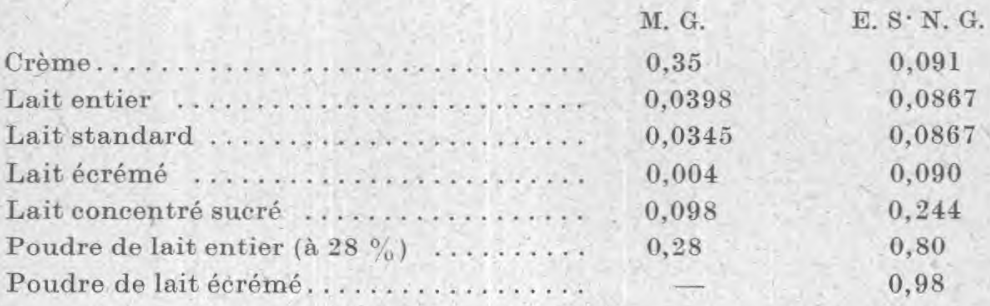

Sans vouloir aller trop loin, il est évident que tous les produits laitiers de l'usine susceptibles d'apporter de l'extrait see non gras et de la matière grasse peuvent être utilisés : babeurre, rinçures de vaccum, etc.

Parmi ces éléments, il y en aura, comme le lait concentré sucré ou le lait standard, qui sont accidentels ; par contre, la crème, les poudres de lait ou le lait entier du jour sont quotidiennement à la disposition du laboratoire.

c) Exemples de préparation.

Rappelons le but à atteindre :

$42 \mathrm{~kg}$. de M.G.

$108 \mathrm{~kg}$. d'E.S.N.G.

$\overline{150} \mathrm{~kg}$. d'extrait sec de lait,

par pétrissée de 600 kilogrammes.

\section{Lait écrémé en poudre et crème.}

C'est le cas le plus simple :

$100 \mathrm{~kg}$. de crème apportent,35 kg. de M.G.

$120 \mathrm{~kg}$. en apporteront $42 \mathrm{~kg}$.

Ces $120 \mathrm{~kg}$. apporteront également :

$$
120 \times 0,091=10 \mathrm{~kg} .92 \text { d'E.S.N.G. }
$$

Le lait en poudre devra apporter le complément :

$$
108-10,92=98,08
$$


Cet extrait sec est contenu dans 100 kilogrammes.

Il est bien évident que pour faire ce mélange dans le pétrin, il y aura lieu de le diluer avec de l'eau.

\section{Lait entier et poudre de lait écrémé.}

Le lait entier apportera la M.G. ; il en faudra :

$$
\frac{42}{0,0398}=1.050 \mathrm{~kg} \text {. }
$$

apportant en plus : $1.050 \times 0,0867=91,035 \mathrm{~kg}$. d'E.S.N.G.

Le lait en poudre devra fournir le complément soit :

$108-91=17 \mathrm{~kg}$. se trouvant dans $17 \mathrm{~kg}$. 7 ou $18 \mathrm{~kg}$. de lait en poudre.

Il est à craindre que la dilution ne soit trop grande, aussi il y a lieu de concentrer le lait entier, ou mieux, comme dans le cas suivant, de l'enrichir avec de la crème.

\section{Poudre de lait écrémé, lait entier, crème.}

La dilution optima, expérimentalement constatée, est de l'ordre de 700 à 800 litres de liquide, de façon à ce que le pétrin contienne 1.000 à 1.200 kilogrammes de produits.

Dans la fabrication suivie, il a été mis 700 litres de lait du jour (M.G. = 3,98\%) par pétrissée.

Ce lait apporte : $700 \times 0,0398=27 \mathrm{~kg} .86$ de M.G.

Pour compléter à 42 kilogrammes, il devra être ajouté $14 \mathrm{~kg}$. 14 de M.G. se trouvant dans :

$$
\frac{14,14}{0,35}=44 \mathrm{~kg} .4 \text { de crème à } 35 \% \text {. }
$$

Ce lait et cette crème apportent l'extrait see non gras :

$$
\begin{array}{rr}
\text { Lait : } 700 \times 0,0867= & 60,69 \\
\text { Crème } 44,4 \times 0,091= & 4,04 \\
\hline 64,73
\end{array}
$$

Pour compléter à 108 , il faudra : $108-64,73=43 \mathrm{~kg} \cdot 27$ d'extrait sec qui sera fourni par 45 kilogrammes de lait en poudre écrémé. Ce mélange est le plus courant et le plus commode:

\section{Lait standard, crème, poudre de lait écrémé.}

Certains jours, tout le lait reçu à la laiterie est standardisé pour la condenserie, à $3,45 \%$, ce qui oblige le chef de fabrication des farines lactées à utiliser ce lait standard pour ses pétrissées.

Dans ce cas, les proportions sont modifiées.

Le volume "commode" pour la fabrication de la "soupe " de lait standard sera de 700 litres. Ce lait apportera : 
$700 \times 0,0345=24 \mathrm{~kg} .15$ de M.G.

Pour compléter à 42 kilogrammes, il devra être ajouté $17 \mathrm{~kg}$. 85 de M.G., soit

$$
\frac{17,85}{0,35}=51 \mathrm{~kg} \text {. de crème à } 35 \% \text {. }
$$

Le lait standard et la crème apportent l'E.S.N.G. suivant :

$$
\begin{aligned}
& \text { Lait standard : } 700 \times 0,0867=60,69 \\
& \text { Crème: } \quad 51 \times 0,091=4,64 \\
& 65,33
\end{aligned}
$$

Pour compléter à 108 , il faudra :

$108-65,33=42 \mathrm{~kg} .67$ d'extrait sec non gras apporté par 44 kilogrammes de lait sec maigre.

\section{Lait concentré sucré, crème et lait.}

La meilleure façon d'utiliser le lait concentré sucré qu'il est impossible de mettre en boîte : lait ayant goût de cuit, de composition défectueuse ou provenant de boîte couleuse, est de s'en servir pour la fabrication de la farine lactée.

Admettons que la composition de ce lait concentré sucré soit la suivante :

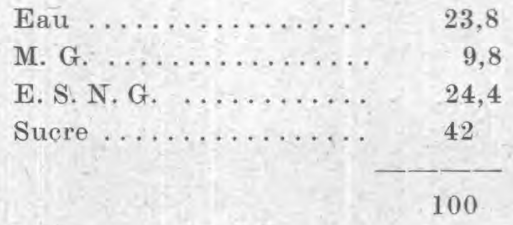

Comme à chaque pétrissée, il doit être apporté 42 kilogrammes de sucre, on utilisera 100 kilogrammes de lait concentré sucré apportant le sucre nécessaire, et on ne sucrera pas.

Ces 100 kilogrammes de lait concentré sucré apporteront 9 kg. 8 de M.G. et 24 kg. 4 d'E.S.N.G.

Le reste devra être fourni par du lait et de la crème soit :

$$
42-9,8=32 \mathrm{~kg} .2 \text { de M.G. }
$$

et :

$$
108-24,4=83 \mathrm{~kg} .6 \text { d'E.S.N.G. }
$$

Or, en admettant que la crème et le lait ont le même E.S.N.G. de l'ordre de $9 \%$, pour avoir 83 kg. 6 d'E.S.N.G., il faut mettre en œuvre : $\frac{83,6}{0,09}=927 \mathrm{~kg}$. de lait ou de crème, qui devront avoir une richesse de $: \frac{32,2}{927}=0,0346$. Par conséquent, du lait à 0,0398 est trop riche en matière grasse. 
Par contre, du lait standard à 0,0345 conviendrait. Il faudra en ajouter 927 kilogrammes soit en arrondissant 930 kilogrammes.

6. Lait concentré sucré, crème, poudre de lait.

Au lieu d'ajouter 927 kilogrammes de lait à 0,0346 , un mélange de crème et de poudre de lait peut être préparé.

La crème apportera la M.G. et il en faudra :

$$
\frac{32,2}{0,35}=92 \mathrm{~kg} \text {. }
$$

apportant : $92 \times 0,091=8 \mathrm{~kg} .372$ d'E.S.N.G. La poudre de lait apportera le reste soit : $83,6-8,372=75 \mathrm{~kg}$. 23 représentés par 77 ou 78 kilogrammes de poudre de lait environ.

Il est bien entendu que de l'eau complètera le mélange.

\section{Poudre de lait entier à $28 \%$ et crème.}

Enfin, il arrive que l'usine doive se servir de poudre de lait entier, d'importation par exemple, ou fabriquée aux périodes de grande production laitière.

Dans ces conditions, le mélange nécessaire par pétrissée, en posant x kilogrammes de poudre de lait à $28 \%$ de M.G. et y kilogrammes de crème à $35 \%$, se calcule en résolvant les deux équations suivantes :

ce qui donne :

$$
\begin{aligned}
& \text { E.S.N.G. : } \quad 0,8 x+0,09 y=108 \\
& \text { M.G. : } \quad 0,28 x+0,35 y=42
\end{aligned}
$$

$$
\begin{aligned}
& x=135 \mathrm{~kg} \text {. de lait en poudre à } 28 \% \\
& y=18 \mathrm{~kg} \text {. de crème. }
\end{aligned}
$$

Là également, de l'eau devra être ajoutée à ce mélange.

D'autres mélanges pourraient être encore imaginés, mais d'utilisation beaucoup moins courante, alors que ceux-ci sont fréquents.

En pratique, ces mélanges sont indiqués dans un barème, avec un coefficient de sécurité suffisant pour compenser les pertes. Mais, l'établissement d'un barème nécessite des matières premières constantes, ce qui est loin d'être le cas avec un ra massage journalier.

\section{Tableau des équivalences}

(pétrin de 1.700 litres, fabrication de $600 \mathrm{kgs}$ de farine lactée)

Kilogrammes

1. Lait en poudre écrémé $\ldots \ldots \ldots \ldots \ldots \ldots \ldots \ldots . . \ldots$

Crème ........................ 120

2. Lait entier $\ldots \ldots \ldots \ldots \ldots \ldots \ldots \ldots \ldots \ldots \ldots \ldots \ldots \ldots \ldots$

Lait en poudre $\ldots \ldots \ldots \ldots \ldots \ldots \ldots \ldots \ldots \ldots . \ldots$

3. Poudre de dait écrémé ............... 45 
Kilogrammes

Lait entier . . . . . . . . . . . . . 700

Crème . . . .

4. Lait standard .................. 700

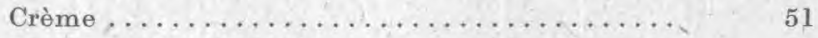

Poudre de lait écrémé ............... 44

5. Lait concentré sucré $\ldots \ldots \ldots \ldots \ldots \ldots \ldots \ldots \ldots . \ldots 100$

Lait standard $\ldots \ldots \ldots \ldots \ldots \ldots \ldots \ldots \ldots \ldots \ldots$

6. Lait concentré sucré ................. 100

Crème .....................

Poudre de lait ..................

7. Poudre de lait entier $28 \% \ldots \ldots \ldots \ldots \ldots \ldots$

Crème..................... 18

\section{ÉTUDE PHYSICO-CHIMIQUE DU LAIT \\ par}

W. KOPACZEWSKI

GELIFIGATION DU LAIT (suite)

\section{B. Gélification du lait par la présure}

\section{HISTORIQUE}

La présure a peu été étudiée au point de vue physico-chimique. Notons tout d'abord, que son individualité a été contestée : pour PAW'LOW, elle serait identique à la pepsine ; la différence entre les deux ferments consisterait uniquement en la liquéfaction du gel provoqué par l'action de la pepsine, alors que celui dû à la présure est irréversible.

Notons aussi, avec DANILEWSKY et SAWIALOW, que la présure peut provoquer la floculation des peptones et des protéoses.

Voici ce que l'on savait sur les caractères physico-chimiques de la présure.

La constante de diffusion a été mesurée par HERzog et KASARNowSKI : elle est analogue à celle de la pepsine et deux fois plus forte $(0,064)$ que celle de la saccharase et de l'émulsine ; celle d'ovalbumine est dans les mêmes conditions expérimentales, de 0,054. En appliquant à ces chiffres la formule proposée par THOverT, le poids micellaire d'ovalbumine serait de 17.000 et celui de la présure de 14.000 .

La présure dialyse à travers les membranes a mniotique (JACOBY); elle est presque totalement arrêtée par les membranes lécithinées (SWART) et ne traverse pas celles de cellulose (VANDEVELDE). 